\title{
Transformaciones de la narrativa del cambio climático global en Uruguay
}

\author{
Javier Taks*
}

\section{Resumen}

Entre 2005 y 2009 el cambio climático global aumentó su presencia mediática en Uruguay y terminó de institucionalizarse a nivel estatal, motivado por la experiencia colectiva de eventos meteorológicos extremos y una estrategia discursiva frente a la agenda internacional que incluyó a la sociedad nacional uruguaya en una humanidad planetarizada y en riesgo frente al cambio climático peligroso de origen antrópico. Este artículo busca, por un lado, mostrar cómo se ha ido transformando la narrativa del cambio climático de acuerdo a la visión hegemónica del desarrollo nacional desde los 1990 al presente. El clima se ha vuelto una arena de lucha de sentidos cuando se reincrustó en las discusiones sobre las formas de producción y consumo. Por otro lado, se describe y analiza la circulación y acomodación cognitiva de dicha narrativa ya nacionalizada en contextos particulares de uso social y político. Los casos a comparar incluyen científicos del clima, trabajadores de la industria energética y pescadores artesanales. En las conclusiones se aborda la brecha entre el uso institucionalizado de la narrativa global del cambio climático y las percepciones del tiempo y clima en la cotidianidad de las personas y cómo, al momento del estudio, no significaba un obstáculo a la reproducción de los modos de vida y producción.

Palabras clave: Institucionalidad climática, Políticas públicas del clima, Calentamiento global, Variabilidad climática, Percepción del tiempo y clima.

* Universidad de la República, Montevideo, Uruguay. 


\section{Transformations of the narrative on global climate change in Uruguay}

\section{Abstract}

Between 2005 and 2009 global climate change increased its media presence in Uruguay and became institutionalized at state level, motivated by the collective experience of extreme weather events and a discursive strategy in the presence of the international agenda that included Uruguayan society in a humanity planetarized and at risk in the face of dangerous climate change of anthropic origin. This article seeks, on the one hand, to show how the climate change narrative has been transformed according to the hegemonic vision of national development from the 1990's to the present. The climate has become an arena of struggle over meanings when it was reintroduced in discussions about the forms of production and consumption. On the other hand, it describes and analyzes the circulation and cognitive accommodation of this narrative already nationalized in particular contexts of social and political use. Compared cases include climate scientists, energy industry workers and artisanal fishermen. The conclusions address the gap between the institutionalized use of the global narrative of climate change and the perceptions of time and climate in people's daily lives and how, at the time of the study, it did not mean an obstacle to the reproduction of ways of life and production.

Keywords: Climate institutionality, Climate public policies, Global warming, Climate variability, Weather and climate perceptions.

\section{Introducción}

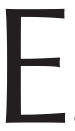

n el año 2009, cuando a nivel global se intentaba lograr un acuerdo internacional para mitigar el calentamiento global de origen antropogénico en la Cumbre del Clima en Copenhague, la "respuesta al cambio climático" se convirtió en política de estado en Uruguay al instaurarse por decreto presidencial el Sistema Nacional de Respuesta al Cambio Climático y la Variabilidad (SNRCC). Un año más tarde, en 2010, a poco de asumir el nuevo gobierno del Frente Amplio presidido por José Mujica, el eje temático "Cambio Climático" fue priorizado por la clase política dentro de la cuestión medio ambiental, avalando el Plan Nacional de Respuesta al Cambio Climático elaborado a fines del 2009 y definiendo que el país usará los recursos propios "en las estrategias de adaptación a la manifestación de fenómenos meteóricos [sic] extremos de la variabilidad 
climática, considerando sus efectos sociales, ambientales y económicos adversos", al tiempo que se comprometía a captar fondos internacionales y de la cooperación internacional también para la adaptación, identificando al sector energético como prioritario por su contribución al cambio climático y los mercados de carbono como instrumento económico potencial.

En apenas dos años, parecía que se había llegado a un consenso nacional, al menos en las elites político-partidarias y de la expertocracia internacional radicada en Uruguay, sobre la importancia del cambio climático para todos los ciudadanos del país; se hacía visible en la "agenda política" un campo de pensamiento y acción tradicionalmente relegado a una muy pequeña burocracia en el estado y a un creciente número de "expertos" uruguayos y extranjeros. ${ }^{1}$ Sin embargo, esta imagen de consenso no clausuró los usos sociales y políticos del cambio climático global, que ha mostrado significativa diversidad sobre las causas y formas de adaptación al cambio y la variabilidad climática. Siguiendo a Milton (2008), se entiende que los desafíos que presentan el calentamiento global y el cambio climático no sólo precisan una comprensión de los procesos climáticos y ecológicos, sino que "requiere[n] entendernos a nosotros mismos, como agentes, como víctimas y, en algunos casos, beneficiarios del cambio [...] sin ese entendimiento, las políticas públicas estarán influidas por la ignorancia y falsos conceptos" (Milton, 2008, p. 58).

Con este desafío, de "entendernos a nosotros mismos" frente al calentamiento global y el cambio climático, se llevó a cabo una investigación de corte antropológico, entre 2011 y 2013², cuya estrategia y resultados se exponen parcialmente en este texto que busca comprender las relaciones entre eventos extremos meteorológicos, la narrativa global del cambio climático y el desarrollo de la política pública del clima en Uruguay en

\footnotetext{
${ }^{1}$ En este sentido, el número de investigadores involucrados en proyectos que de una u otra manera se relacionan con el cambio climático son alrededor de 500, de acuerdo a un relevamiento universitario de 2011, lo cual no es despreciable y muestra el aumento de interés en al menos un lustro (García Cartagena; Torranza; Torres, 2013).

${ }^{2}$ El proyecto "Desarrollo de una metodología para el estudio de la percepción cultural del clima en Uruguay en el marco del Plan Nacional de Respuesta al Cambio Climático" fue financiado por Comisión Sectorial de Investigaciones Científicas de la UdelaR entre los años 2011-2013. El equipo de investigación estuvo integrado por los antropólogos Javier Taks, Carlos Santos, María Fernanda de Torres y la psicóloga social Alicia Migliaro.
} 
la primera década del siglo XXI. A su vez, mostrará que cierto consenso alcanzado entre las elites del estado y la academia acerca de las causas, impactos y formas de adaptación al cambio y a la variabilidad climática no está exento de discusiones técnicas y comunicacionales. Para eso se analizaron los procesos de producción de conocimiento científico del clima en la Universidad de la República (UdelaR), la principal institución para la investigación y formación terciaria en el país.

Se señala y analiza, en la tercera sección, que los acuerdos oficiales no se traducen mecánicamente en la vida cotidiana de grupos sociales que viven el tiempo y piensan el clima en sus tareas productivas, como son los pescadores artesanales de la Laguna Merín en la región este y los trabajadores embarcados que operan la boya petrolera de la empresa estatal energética ANCAP en aguas profundas frente a las costas del centro turístico de Punta del Este. Finalmente, en la sección de conclusiones se da cuenta de cómo la hegemonía del progresismo en política económica, entrelazada con una particular visión de la relación sociedad-naturaleza, ha llevado a la moderación de los discursos que veían en el cambio climático la manifestación de la contradicción última entre las fuerzas del mercado capitalista y su naturaleza externa, así como a la moderación de los discursos cornucopianos y escépticos que no ven nada en el cambio climático que necesariamente limite el aumento constante de la producción y el consumo en un marco desarrollista.

\section{Estrategia de investigación}

La aproximación al tema de la circulación de la narrativa global del cambio climático y su vínculo con la política pública fue de naturaleza cualitativa, sistematizando material de prensa y llevando adelante observación participante en sitios de trabajo científico y de producción material, en instancias de discusión técnica y política, así como entrevistas a interlocutores calificados.

Inicialmente se produjo una cronología comparada del siglo XX y XXI que contempla tres variables: eventos climáticos extremos a nivel nacional, eventos climáticos extremos internacionales, la institucionalidad internacional del cambio climático (cumbres, protocolos, eventos culturales) 
y la institucionalidad climática nacional (organismos, leyes, proyectos). La información de base fue la prensa escrita: semanario Brecha, suplemento de energía de la diaria y artículos de prensa del archivo del Centro Uruguayo de Tecnologías Apropiadas (CEUTA) que incluye semanario Búsqueda y los diarios El País, El Observador y La República.

Por otra parte, se sumó a la cronología el análisis de documentos públicos de autores y organismos clave encontrados en sitios web y bibliotecas públicas y privadas, lo que permitió delinear un mapa de actores y sus discursos dominantes en relación al cambio y la variabilidad climáticas, el efecto invernadero, el desarrollo económico y la protección ecológica, entre otros. A lo cual se agregaron entrevistas en profundidad a interlocutores de la Unidad de Cambio Climático (UCC) del Ministerio de Vivienda, Ordenamiento Territorial y Medio Ambiente, del Sistema Nacional de Respuesta al Cambio Climático (SNRCC), del CEUTA y del Centro Interdisciplinario de Respuesta al Cambio y la Variabilidad Climática (CIRCVC) de la Universidad de la República.

A partir de contar con una visión de cómo evolucionó la narrativa del cambio climático, el equipo realizó trabajo de campo en tres situaciones etnográficas: a) un laboratorio de científicos del clima del Instituto de Física de la Facultad de Ciencias y en el Instituto de Mecánica de los Fluidos (IMFIA) de Facultad de Ingeniería; b) una zona de pesca artesanal en la cercanía del Balneario Laguna Merín y área de influencia, en el Departamento de Cerro Largo al noreste del país; y c) el territorio marino de los capitanes de amarre y trabajadores de la Boya Petrolera de José Ignacio, propiedad de la empresa estatal petrolera ANCAP. La duración del trabajo de campo fue diversa, pero en un rango de 6-8 meses.

Otra forma de recolección de información fue la organización de mesas de discusión con expertos en meteorología y ciencias del clima.

\section{Reincrustación del cambio climático en el estado}

El mapa de actores en torno a la narrativa del cambio climático muestra que es en la esfera estatal y, en particular, en el Poder Ejecutivo donde ésta se ha institucionalizado, desde el cambio de siglo y hasta 2013, con mayor continuidad y de forma trasversal. En el resto de los sectores de la 
sociedad uruguaya - empresarial, sociedad civil, movimientos sociales - no hay densidad ni masa crítica en el tema, con una creciente importancia a nivel de la investigación académica. Probablemente esto responda a una mutua alimentación entre generación o sistematización de conocimientos y el establecimiento de políticas y programas públicos sobre el cambio climático, la mayoría de los cuales depende en su origen y en gran parte de su desarrollo de la cooperación internacional.

Por otro lado, los eventos extremos que registran la memoria colectiva y la historia provienen del siglo XIX y es la "sequía" la gran protagonista. Lo cual parece lógico en un país de base ganadera, tan dependiente de las pasturas que, a su vez, son fuertemente dependientes de la humedad del suelo y las precipitaciones. Luego, a mediados del siglo XX, aparecen las "inundaciones del 59" como mojón ineludible en la relación entre organización social y eventos meteorológicos a nivel nacional, dando mayor visibilidad a la problemática urbana.

Es importante notar que, hasta mediados de la década del 90 del siglo pasado, los fenómenos climáticos extremos o extraordinarios no parecen asociarse de ninguna manera al cambio climático global; simplemente se constataban como parte de las características del clima en el país. Así lo demuestra el hecho de que el seminal Estudio Ambiental Nacional, coordinado por la Organización de Estados Americanos y publicado en 1992 en preparación de la representación oficial uruguaya en la Cumbre de Rio, no menciona en ningún momento el término "cambio climático" (OPP/BID/ OEA 1992). Muy distinta es la situación del informe GEOUruguay 2008, de similar alcance descriptivo, que cuenta con al menos cuatro secciones en cuyo título aparece el cambio climático (CLAES/PNUMA/DINAMA, 2008). Esto se vincula a que, junto con el aumento de la comunicación de los eventos meteorológicos extremos, no solo del país sino también en otras partes del mundo, se da un proceso paralelo, desde 1992, de institucionalización oficial del cambio climático global a partir de la firma y posterior ratificación de la Convención para el Cambio Climático.

Uno de los eventos extremos climático-políticos clave que han surgido en nuestra investigación es el temporal del 23 de agosto de 2005, un "ciclón extra-tropical" no previsto por el servicio meteorológico público, 
que ocasionó una muerte humana, la muerte de ganado y destrozos materiales. Podemos afirmar que aquella tormenta actuó como catalizadora de una política pública: la creación del Sistema Nacional de Respuesta al Cambio Climático; el reimpulso para una Licenciatura en Ciencias de la Atmósfera en la UdelaR, y la necesidad de fortalecer a la ex Dirección Nacional de Meteorología del Ministerio de Defensa, hoy convertida en Instituto Uruguayo de Meteorología vinculado al Poder Ejecutivo a través del MVOTMA (Ley n 19.158 del 23 octubre de 2013).

Encontramos una relación entre fenómenos percibidos del tiempo, la narrativa del cambio climático global (relativizada por muchos científicos locales) y las políticas públicas que, en general, la usan, aunque no la asumen totalmente en las acciones. Pero la relación no es mecánica, depende de los eventos extremos y los procesos de institucionalización del clima en la interfase global y nacional. La circulación de imágenes mundiales de inundaciones, sequías, tormentas, olas de calor, incendios, y derretimiento de hielos - fenómenos todos que son de alguna manera asociados en los medios con la narrativa del cambio climático global - se conjuga con las informaciones de inundaciones de ciudades y campos del Uruguay, abriendo así una oportunidad de diálogo y traducción mutua entre científicos y políticos (Latour, 2012) para reincrustar el cambio climático en la política y en las políticas públicas. Pues, mientras la ciencia objetiva y externaliza el clima, los distintos actores políticos lo intentan reincrustar en la sociedad de acuerdo a sus necesidades e intereses coyunturales. Veamos dos ejemplos.

El ex ministro de agropecuaria, Tabaré Aguerre, productor arrocero y vocero de la realidad del "campo", con frecuencia habló sobre los proyectos y programas de su Ministerio volcados a mitigar la vulnerabilidad de los productores rurales ante la incertidumbre climática, en particular los ganaderos. Sin embargo, para su contextualización afirmaba: "En Uruguay el cambio climático no existe, es una moda. Lo que existe es la variabilidad climática." (Seminario FAO, marzo 2012). Sus palabras representan una de las principales ideas de resignificación de la narrativa del cambio climático global para Uruguay: es más importante la variación climática decadal y anual que las tendencias globales, para entender y actuar en las políticas 
públicas agropecuarias de adaptación climática. Se basaba, para construir este supuesto orientado a la praxis, en estudios realizados, por ejemplo, en el IRI de la Columbia University (Baethgen, 2007). El mensaje es que el clima siempre ha afectado en mayor o menor medida la producción material, acompañado de fenómenos hidrometeorológicos extremos, definidos en relación con la variabilidad climática normal. No hay catastrofismo, ni tampoco necesidad de escepticismo o negacionismo del cambio climático, más allá de ser caracterizado por el ministro como "moda". Es un discurso normalizador, no rupturista, que contrasta, de tanto en tanto, con los enunciados de buena parte de los agentes económicos privados del sector agropecuario, que usan el cambio climático como un idioma para obtener mayor atención, mientras reclaman al estado durante sequías o inundaciones. Si fuera un estado normal de las cosas, no se justificaría el reclamo, por el contrario, deberían los agentes económicos tomar las previsiones bajo su responsabilidad; por ejemplo, incluir la variable climática en su matriz de toma de decisiones y contratar seguros agropecuarios.

Un segundo ejemplo acerca de la reincrustación nacional del cambio climático como narrativo global lo presenta la expresión de un antiguo ministro de Medio Ambiente, Carlos Colacce, durante la cumbre de Copenhague, la cual se acerca a la llamada "posición país" (Lattanzio; Marrero, 2012) en el marco de las relaciones internacionales del clima: “Necesariamente, nuestra prioridad es la adaptación. (...) Dentro de nuestras capacidades económicas estamos realizando los mayores esfuerzos para ello. Por ello es ineludible e impostergable, tal como la Convención [Marco del Cambio Climático] lo establece, la cooperación internacional a efectos de obtener los recursos y la tecnología necesaria." En esta significación de lo climático, la escala nacional es jerarquizada con relación a lo global. Por ello se repite la autoidentificación de "pequeño país", que no es responsable por emisiones de GEI, pero sí es afectado por los efectos del cambio climático y, por lo tanto, necesitado de recursos externos para la adaptación. Los datos objetivos justificarían dicho posicionamiento. Uruguay tiene casi 3.300.000 habitantes humanos y un poco más de 11 millones de cabezas de ganado vacuno. En el año 2014, las emisiones totales netas de GEI, medidas usando el GTP ${ }_{100}$, fueron 28.341 Gg CO2 eq, lo que representó el 
0,06\% de las emisiones mundiales de GEI antropógenos, siendo los sectores agropecuario (principalmente ganadería) y de la energía (principalmente transporte) las principales fuentes de GEI (MVOTMA, 2017, p. 19). Asimismo, desde el comienzo del siglo XXI hasta 2009 ha sufrido dos importantes inundaciones que produjeron 30.000 evacuados y pérdidas económicas no menores a 30 millones de dólares. Al menos dos sequías impactaron al país en este mismo período estimándose un perjuicio económico mínimo de 1.000 millones de dólares (Lattanzio; Marrero, 2012). Finalmente, en 2011-2012 la Cooperación Internacional para el sector Cambio Climático aportó 20 millones de dólares, la mitad para la adaptación del sector agrícola a los déficits hídricos, y la otra mitad para la promoción de las energías renovables, representando un tercio de toda la cooperación internacional al estado uruguayo (AUCl, 2012).

Así como más arriba señalamos que una de las formas de reincrustar el cambio climático global en la política oficial era normalizándolo a través de la variabilidad climática (aunque acentuada indudablemente en sus extremos hidrometereológicos), en el segundo ejemplo, la reincrustación se da priorizando, casi exclusivamente, la adaptación a un cambio que ha sido generado fuera de las fronteras nacionales.

\section{La mirada de los científicos del clima}

Para ser un científico del clima se debe ubicar las relaciones físicas en el sistema del clima, que vincula la atmósfera, los océanos y las características de la tierra. Mientras en la Facultad de Ciencias de la Universidad de la República los científicos se vinculan con estudios básicos sobre la relación del clima con otras dimensiones de la vida, como por ejemplo, la biodiversidad en ecosistemas terrestres y acuáticos o el cambio de uso del suelo, en la Facultad de Ingeniería buscan la aplicación concreta de su conocimiento acerca del comportamiento del clima para la predicción en actividades estratégicas como generación de hidroenergía o la navegabilidad en mares y ríos. Existen entonces algunas diferencias en la comunicación entre los científicos de clima, según estos dos principales perfiles, y aquellos quienes buscan respuestas para la fabricación de política pública: 
[S]oy un 'climate scientist', lo que se trata es de avanzar el conocimiento en torno a cómo funciona ese sistema climático en torno a distintas escalas temporales, tres meses, la variabilidad interanual, decadal, y allá lejos el cambio climático. Es tratar de entender cómo funciona físicamente ese sistema: atmósfera, océanos y suelo, a una escala espacial más grande (Entrevista 1) (énfasis agregado).

La Ingeniería, a diferencia de la investigación básica, cultiva en su perfil dar respuestas concretas a problemas de la realidad. En el caso particular de la ciencia del clima, los científicos buscan ubicar dicha disciplina en un lugar de reconocimiento público a través de la utilidad social, productiva y estratégica del conocimiento que producen, por ejemplo, midiendo impactos:

[Existe] una presión insostenible del medio, empezamos a virar hacia el tema cambio climático, aunque yo no podría decir que mi producción científica es en cambio climático (...) tengo una vocación muy clara de intentar aportar a la construcción de que se manejen mejor estos temas en el Uruguay (...) (Entrevistado 3) (énfasis agregado).

Entonces, parece existir una principal diferencia entre los científicos del clima (básicos) y los otros científicos abocados a las mediciones de impacto del cambio y la variabilidad climática. Dicha diferencia se relaciona con la definición y trato acerca de los escenarios futuros. Mientras que, para los científicos básicos del clima. los escenarios son probables pero no ciertos, quienes estudian impacto, y por lo tanto centran su foco de atención en otras relaciones, dan por dado un tipo de paisaje futuro - más o menos lluvias, sequías, temperatura - y se adentran a sus variables y dimensiones propias.

[G]eneralmente la gente que trabaja en impacto da por hecho un montón de cosas, y la gente que trabaja en básica, dice 'cuidado', y ahí empiezan los problemas (risas). Yo entiendo. Si querés hacer un estudio de impacto iqué tomas como base? Entonces, en general, los trabajos de impacto son bastante complicados porque el tema de la incertidumbre es muy complicado para hacerlo bien, y en general la parte de la incertidumbre se deja de lado para hacer otra cosa: 'este va a ser el impacto' y listo. En general se pone una 
frase al comienzo que dice algo así como 'estos resultados del IPCC tienen incertidumbre, pero vamos a tomarlos como base y seguimos', una especie de disclaimer al principio, luego se olvida (Entrevista 2).

A su vez, los científicos del clima y los no expertos (tomadores de decisión, productores y técnicos agropecuarios, periodistas, entre otros) sostienen una diferencia sobre la comprensión de la incertidumbre. Para los expertos, la incertidumbre es una característica del mundo material, la realidad es incierta, mientras que los no expertos interpretan la incertidumbre como una medida de la ignorancia (ver Taddei, 2012). Por ello la creciente demanda social y política sobre los meteorólogos y científicos del clima para la generación de pronósticos ciertos. El cambio climático, tal cual circula en la televisión y redes sociales, asociado a imágenes de paisajes de desastres por inundación, sequías, nevadas o tornados, provoca aún más confusión en la comunicación entre científicos y legos:

El tema de asociar catástrofes a cambio climático no sé por qué lo hacen, va más allá de mi comprensión. Porque si uno le llama catástrofes a la existencia de huracanes o la existencia de un tornado, eso siempre existió. ¿A qué le llamamos catástrofe? Digamos entonces, no es ni siquiera una proyección de que vaya a haber más huracanes o nada, no está claro eso. El problema es cómo se maneja esa información. Yo entiendo la agenda atrás, pero donde no se cumpla algo de eso, qué hacés. Entiendo también que la gente tiene poca memoria y vos podés jugar con eso. Entiendo también que si no pones un oso polar arriba de un témpano derritiéndose la gente no comprende el problema, y haga algo al respecto. Pero hay una línea muy fina entre hacer eso y mentirle a la gente, sobre todo cuando uno mira las bases para hacer estas afirmaciones, aumenta el problema. Quizás me diga que es sobre la base de unos papers, pero quizás existan otros papers que digan cosas contrarias (Entrevista 2).

Un aspecto que nuestra investigación apenas hizo emerger es cómo la narrativa del cambio climático global penetra en nuestro país y en la comunidad de científicos del clima a partir de las relaciones con los organismos globales. Para nuestro caso, la Organización Meteorológica Mundial (OMM) es clave, referida por muchos entrevistados como el "Vaticano del Clima". La OMM dicta las políticas de educación en 
meteorología, así como los requerimientos de conocimiento para ocupar lugares estratégicos de trabajo (por ejemplo en los aeropuertos); coordina y articula centros de investigación, entre otras funciones. Es la referencia oficial del clima, donde la integración se caracteriza por cuerpos técnicos, más que políticos, que lideraron la articulación del primer IPCC. Si seguimos la analogía con el Vaticano, la "religión meteorológica" tiene una jerarquía bien clara y unas oraciones a repetir. Quizá en el futuro se pueda pesquisar este vínculo global-local a partir de la descripción de la formación de comunidades epistémicas específicas (ver García Cartagena; Taks, 2015, para un caso de intercambio académico sobre gestión costera y cambio climático).

\section{Tiempo y clima entre petroleros y pescadores}

Una de las áreas sensibles a los cambios en el clima de la empresa nacional de petróleo ANCAP es la boya petrolera ubicada frente a José Ignacio en la costa oceánica del país, única puerta de entrada en escala industrial del crudo con el que funciona la refinería de La Teja, en Montevideo. A través de la boya ingresa todo el petróleo crudo importado, unos 16 millones de barriles por año, lo que representa el amarre de un super-petrolero cada 22 días, aproximadamente. La boya petrolera está conectada a la Terminal del Este y a sus tanques de almacenamiento por un oleoducto subacuático de casi 4 kilómetros de largo; desde los tanques de almacenamiento se bombea el petróleo hacia la refinería en la capital del país a unos 180 km de distancia.

La afectación a partir de la variabilidad climática es indiscutible. Según datos de la Gerencia de Logística y Operaciones, en los 38 años de funcionamiento del sistema, la ventana anual de operaciones se redujo del $50 \%$ a un nivel que se encuentra entre el $35 \%$ y el $30 \%$. Esta ventana de operaciones se define como los días en los que efectivamente se puede operar en el año para la transferencia de petróleo de los barcos a la boya. Las condiciones seguras de operación dependen de la altura de las olas, la velocidad del viento y la fuerza de las corrientes marítimas. El 98\% de las causas de las demoras de operación en la Boya corresponde a las 
condiciones climáticas adversas, que difícilmente se pueden prever más allá de tres o cuatro días.

El equipo de trabajo para las operaciones de amarre es de unas 30 personas, todos hombres, liderados en 2013 por tres capitanes de amarre (mooring masters), los cuales tienen formación en la Armada Nacional y cursos de especialización en el extranjero. De acuerdo a estos capitanes, lo que más se constata en los últimos diez años es una pérdida de la estacionalidad y el aumento en frecuencia de las "líneas de inestabilidad" que confunden aún más lo que de por sí es entendido como una "zona del mar complicada", pues se enfrentan corrientes subacuáticas muy cambiantes a lo largo del día. En la descripción de la relación de su tarea con las condiciones meteorológicas y las transformaciones climáticas enunciadas, los capitanes incorporan en todo momento el contexto social, político y económico en su definición de "zona complicada", teniendo una lista clara de los factores que generan una presión externa sobre su operación: la necesidad de salvaguardar la seguridad de los trabajadores, que son una mezcla de empleados asalariados de ANCAP, buzos contratados del servicio de buceo de la Armada Nacional y trabajadores independientes tripulando remolcadores; la prevención de cualquier derrame de petróleo frente al principal centro turístico del país; el costo que significa para ANCAP cada día que un barco petrolero no puede descargar (estimado en 40 mil dólares); y la presión de los capitanes de los petroleros, que reciben a su vez la demanda de sus empresas, por salir a buscar su próximo cargamento so pena de tener que pagar multas por retraso.

La toma de decisiones para las operaciones de amarre y desamarre es colectiva, cada uno desde su función, aunque la última palabra la tienen los capitanes de amarre quienes sintetizan los factores para la buena decisión en "programación, olfato y suerte". No pocas veces significa negociaciones y discusiones con los capitanes del petrolero acerca de las condiciones meteorológicas y la necesidad o no de desamarrar para evitar un choque del barco contra la boya. Lo mismo con los gerentes de ANCAP en Montevideo, con quienes, a partir de la introducción de computadoras en la operativa, hay posibilidades de mayor coincidencia en la interpretación del estado del tiempo local. 
El tiempo y la atmósfera son una presencia ineludible en la vida laboral de los capitanes de amarre y del resto de los trabajadores de la boya petrolera. Flotando en medio del mar, con un gran horizonte abierto de 360 grados, no parece fácil objetivar los componentes del "clima". Todo parece atravesarlo a uno cuando está fuera de la cabina. Dentro de ésta la situación es otra; radares, sonares y distintos aparatos electrónicos dan información no directamente perceptible, útil para mejor orientarse y evitar siniestros. La netbook conectada a un modem inalámbrico permite navegar en Internet e ir chequeando en windguru la información meteorológica, así como seguir un partido de fútbol de la tarde. Los cambios del tiempo, pero también de las relaciones humanas, se afrontan en lo cotidiano sin mayores complejidades, aunque es creciente la preocupación, al menos de los capitanes de amarre, por una mejor adaptación de la tarea a condiciones meteorológicas novedosas, que no se asocian con el cambio climático.

Es que, en relación con las manifestaciones de cambios recientes en el clima, los capitanes de amarre se afilian a la idea de que las causas de estos cambios se refieren a ciclos naturales de la variabilidad climática. Dichos ciclos, de 30 a 40 - según su opinión - responden a la actividad solar. La hipótesis surge de las exposiciones públicas sobre el tema de su meteorólogo de confianza. Dicha confianza climática se funda en el hecho de que el meteorólogo en cuestión tiene formación militar en la misma arma que los capitanes de amarre, ha navegado y conoce la zona de la boya $y$, de acuerdo con nuestros interlocutores, sus pronósticos han acertado. ${ }^{3}$

Es necesario mencionar, no obstante, que en casi todas las instancias de conversación con los trabajadores, técnicos de planta y marineros, la duda en torno a las causas del cambio climático era casi generalizada, no así el reconocimiento de sus manifestaciones; es decir, la categoría "cambio climático" está presente, su significado general de transformación es compartido, pero aún es una arena abierta de sentidos en cuanto al lugar de la acción humana vis-a-vis el clima externo. La respuesta a nuestra

${ }^{3}$ La misma explicación del cambio climático por efecto de los ciclos solares aparece en la obra de un conocido gestor ambiental (Latchinián, 2011), quien fuera además exdirector nacional de medio ambiente (2002-2004) y ex integrante del directorio de ANCAP (2008-2010). Como vemos, la narrativa del cambio climático global a lo IPCC no está tan consensuada en Uruguay. 
interrogante sobre la meteorología y posibles variaciones se convertía, en pocos instantes, en una contra-pregunta acerca de nuestro conocimiento u opinión. A partir de nuestra respuesta, de que para nosotros, como investigadores sociales en el proyecto universitario, el cambio climático existía desde el momento mismo que la gente habla sobre él, la reacción era de apertura a conversar temas de política climática, oportunismo personal, posibles manipulaciones y, en contra de la visión entre los capitanes de amarre, la responsabilidad de la emisión antropogénica.

Más allá de esta tensión entre explicaciones nativas, en diálogo con conversaciones globales, la empresa de petróleo y las personas enfrentan las variaciones climáticas a través de incorporar tecnologías y nuevos modos de gestión. Las principales medidas han sido mejorar los instrumentos de medición en la boya, con miras a contar con una línea de base más confiable y actualizada; también comprar repuestos de la boya de la mejor calidad que disminuyan las tareas de mantenimiento; al mismo tiempo acelerar las operaciones de mantenimiento con mejores herramientas, en vista de que hay menos días aptos para trabajar. La expectativa a futuro es de un aumento del número de barcos que amarrarán en la única boya del país, con lo cual la incidencia del clima será creciente en los resultados prácticos y económicos.

A unos $300 \mathrm{~km}$ de distancia de José Ignacio, hacia el noreste, se encuentra el Balneario Lago Merín, a orillas de la Laguna Merín, en la frontera entre Uruguay y Brasil, a $12 \mathrm{~km}$ del puerto de Río Branco. Allí habitan la laguna, entre otros, los "pescadores artesanales". ${ }^{4}$

Es frecuente encontrar que la pesca es un oficio más, no exclusivo ni excluyente, entre otras prácticas que los sujetos desarrollan, muchas de ellas en relación directa con el ambiente y la extracción de bienes naturales - cazadores, monteadores, apicultores, areneros. La navegación

\footnotetext{
${ }^{4}$ Según el último censo de pesca artesanal, de 2010, se registran un total de 28 embarcaciones en el puerto de Río Branco y su área de influencia (MGAP-DINARA, 2009). Según datos oficiales, en la temporada 2012 se registraron solamente 9 embarcaciones, de las cuales no todas desarrollaron actividad durante la totalidad de la zafra. Las principales especies explotadas en la zona son tararira, bagre y pejerrey (MGAP-DINARA, 2009). El consumo de estos productos en el mercado local es muy escaso, siendo la mayor parte vendido a acopiadores uruguayos y brasileños.
} 
en la Laguna Merín es la base de un conocimiento detallado de "ella": montes, islas, bocas de arroyos, lagunas, bancos de arena, corrientes, que se suman a las anécdotas sobre el trabajo que dejan ver las habilidades en el manejo de los recursos - dónde se facilita u obtura la navegación, las horas de traslado con y sin carga, donde hay que tener cuidado por la baja profundidad, donde se sienten más los azotes del viento, entre otros. Los pescadores son sujetos hábiles y competentes en el manejo del territorio y sus bienes naturales, de los mercados locales y de los contactos, lo cual les da plasticidad para desempeñarse en diversas tareas erigiendo su herramienta de trabajo más preciada: su cuerpo.

Los cambios que, entienden los pescadores, han ocurrido en la Laguna Merín se relacionan con la disminución de la cantidad de pescadores en los últimos años, con la salida de la actividad de los "chicos". Estos pescadores de baja especialización no cuentan con el equipo tecnológico en sus barcas necesario para adentrarse en la Laguna y, al prohibirse por norma estatal la pesca con redes de enmalle en ríos internos, la actividad ya no rinde y la tendencia es a abandonar la pesca, disminuyendo la competencia por el recurso. Otro elemento en que hay opiniones coincidentes es en el aumento de la contaminación de las aguas, aunque difieren en los responsables y valoraciones de los daños. Para la mayoría de los interlocutores pescadores, la contaminación es asociada con las arroceras - producción hegemónica a nivel territorial y el uso extendido de agroquímicos.

Se destaca que el cambio climático como significante está muy poco presente. De hecho, solo un pescador se remite a él y de forma bastante difusa, como un elemento más de degradación ambiental:

- A mí no me gusta cosas que veo, los limos, los venenos, el cambio climático.

- ¿Que opinás del cambio climático?

- A mi entender puede ser parte de la naturaleza, pero mucho tiene la culpa el hombre. Los vientos esos que vienen no son normales. Esos tornados (...). Esos son los daños de la capa de ozono (Entrevistado 5). 
Por otra parte, la única referencia explícita al cambio climático en el territorio de la Laguna Merín la encontramos en la fundamentación de la creación de una Reserva Privada "Bañados del Yacaré" por parte de un empresario de servicios de fumigación aérea. Sobre las costas de la laguna y en algunas islas en la desembocadura del río Tacuarí, se plantea la creación de un "parche de conservación de biodiversidad en tierras productivas (...) como una medida de adaptación al cambio climático" (Arballo, 2011). Sin embargo, la persona que impulsó la creación de esta reserva - que se autodefine como conservacionista - no asegura que exista el cambio climático y menos aún que la responsabilidad sea del hombre. Más aún, en su opinión, la transformación reciente del entorno se debe a la falta de controles públicos, el extractivismo de la pesca artesanal y los malos hábitos de los turistas en el manejo de sus residuos. En otras palabras, la narrativa del cambio climático global parece ser ajena al mundo de sentidos de los habitantes de la Laguna Merín.

\section{Conclusiones}

En Uruguay, en la primera década del actual siglo, era fuerte la idea de que el cambio climático global no entraba aún en la matriz de decisiones del Estado, ni tampoco de los tomadores de decisión del sector empresarial privado. Así lo expresaban funcionarios de la Oficina de Planificación y Presupuesto en un informe interno, tratando de identificar los forzantes del desarrollo nacional en escenarios de mediano plazo:

Por el momento, más allá de manejar grandes titulares, la importancia [del cambio climático] y sus impactos o potencialidades para los sectores productivos es todavía materia casi exclusiva de los especialistas en el mismo (apud Lattanzio; Marrero, 2012, p. 45-46).

Sin embargo, de acuerdo con nuestro estudio, entre 2005 y 2009 , a nivel nacional, el cambio climático se instaló e institucionalizó como un fenómeno explicativo de la relación entre el mundo-globo y el Uruguay, a partir de la combinación, por un lado, de la experiencia colectiva de eventos meteorológicos extremos (por ejemplo, un ciclón extratropical y 
una sequía a continuación) y, por otro, la oportunidad para el gobierno de incluir el país en una humanidad globalizada y en riesgo, definiendo un "nosotros" ambiguo que considera el cambio climático de forma liviana. Es decir, por un parte, la sociedad uruguaya estaría integrada a dicha humanidad globalizada, homogénea, actuando como bloque en la lucha contra la Naturaleza externa, y por otra, esa misma sociedad sería miembro de una parte débil y provincial de una humanidad-otra, una nación víctima de los intereses de los países más desarrollados y poderosos.

Nuttall (2008) analiza, para el caso de Groenlandia, un proceso similar de construcción simultánea de un relato de victimización frente al cambio producido por otros y un relato de oportunidad desarrollista, a partir de los efectos del cambio climático. Pues la pérdida, a causa del calentamiento global, de parte de la capa de hielo de la mayor isla del planeta significa, por un lado, el fin de tradiciones locales Inuit de caza, pesca y recolección - con sus símbolos y significados asociados. Por otro lado, y de acuerdo a autoridades también Inuit, este cambio ambiental se presenta como inmejorable chance de apertura a inversiones extranjeras de multinacionales para la explotación de yacimientos minerales, de energéticos fósiles y la producción hidroeléctrica. Lo más relevante, sin embargo, para pensar el caso de Uruguay y de como el cambio climático pasa a formar parte de las relaciones sociales, es el análisis que hace Nuttall de los estrechos vínculos entre esta ambigua manipulación de la narrativa del cambio climático y el proceso de construcción del Estado-nación autonómico o independiente del reino de Dinamarca. Para lograr ese desarrollo político, las elites gobernantes, también Inuit, promueven un modelo desarrollista de explotación de los nuevos recursos naturales en manos de multinacionales, como la única forma de romper con la dependencia económica danesa. Que, si bien se presenta como una posibilidad de éxito para todos los habitantes, en realidad invisibiliza a los más que probables perdedores de esta apropiación de los comunes: las pequeñas comunidades locales que aún derivan su sustento del acceso a los bienes comunes naturales. En otras palabras, las transformaciones y usos políticos de la narrativa del cambio climático no pueden entenderse por fuera de la continua formación de los estados nacionales. 
El análisis de la trayectoria de la narrativa del cambio climático en Uruguay, desde el 2000 al presente, a nivel de los organismos públicos y la Academia, muestra que ha habido un desplazamiento desde las instituciones ambientales, como el Ministerio de Vivienda, Ordenamiento Territorial y Medio Ambiente y los programas de estudio de las zonas costeras y la biodiversidad, hacia una sectorialización mayor del cambio climático principalmente en la producción agropecuaria y la gestión energética. En el caso de la producción agropecuaria, se usa el concepto vago y multifacético del cambio climático como forma de articular con las tendencias de la cooperación internacional, pero en términos científico-operacionales se realiza un "downscaling cultural" centrado en la variabilidad climática de más corto plazo como fenómeno ya conocido por los expertos y productores, que no provoca escenarios de gran ruptura con los sistemas productivos vigentes. El debate sobre la reciente reforma de la Ley de Riego y su aprobación parlamentaria en 2017, donde la variabilidad climática fue uno de los fundamentos esenciales para su promulgación, muestra justamente como la narrativa del cambio climático puede ser adaptada para acentuar las formas dominantes de producción en el marco de la llamada intensificación sustentable (Santos, 2017). Para el caso de la gestión energética, el cambio climático se usa de forma retórica como justificación para orientar una transición de la matriz energética nacional hacia un menor uso de petróleo crudo. Más que por razones éticas de corte ecológico o social, por argumentos de soberanía nacional (Bertoni et al., 2010).

Los estudios de caso de los trabajadores de la boya petrolera y los habitantes de la Laguna Merín muestran que el nivel de objetivación del cambio climático es mucho más débil en las explicaciones de la realidad cotidiana de lo que aparece en las enunciaciones de funcionarios estatales, consultores e investigadores vinculados directamente a las prácticas burocráticas y la investigación del cambio climático. La percepción integral del tiempo atmosférico y su incrustación en las prácticas laborales es más determinante de las acciones individuales y colectivas que cualquier externalización conceptual del clima o del cambio climático.

Por el momento, no hay grandes contradicciones entre estos dos niveles de concebir y actuar en relación a las condiciones meteorológicas 
y climáticas. A diferencia de otras realidades a nivel internacional (Roncoli; Crane; Orlove, 2009), en Uruguay aún no se han limitado o excluido prácticas económicas y sociales entre los actores locales en nombre del "cambio climático". Esto coincide, por el momento, con la idea de Taddei (2017) de que, a diferencia de otros temas ambientales donde se han impuesto modos específicos de comportamientos (por ejemplo en el uso del agua, la gestión de residuos, bienestar animal o la prohibición del transporte individual en ciudades con el aire contaminado, entre otros), "não há como aplicar essa mesma governabilidade imposta à percepção coletiva a respeito de fenômenos climáticos ou ao uso de previsões climáticas" (Taddei 2017, p. 78; énfasis original).

Más allá de titulares de prensa y nombres de seminarios, o exposición de motivos de leyes, planes y programas, las personas van viviendo y enfrentando de la mejor manera posible las transformaciones graduales y extremas del clima, incrustándolas en su tiempo social y en su taskscape, o paisaje de tareas (Ingold, 2000); para ello, frente a viejas y nuevas incertezas, las personas hacen cotidianamente un "uso criativo das [...] pequenas certezas provisórias" (Taddei 2017, p. 92) que permite mejor sintonizar con fenómenos climáticos novedosos.

Los amarradores de petroleros, los habitantes pescadores de la Laguna Merín y muchos científicos del clima no necesitan la narrativa del cambio climático para explicar sus pequeñas victorias en conciliar con sus habilidades las incertezas del tiempo, el mar, el mercado energético, las inercias organizacionales, la búsqueda de financiamiento y la carrera académica. Tampoco, aún, para entender su lugar en la sociedad y reclamar derechos.

Javier Taks es antropólogo, docente e investigador de la Universidad de la República (UdelaR), Montevideo, Uruguay. Coordinador de la Cátedra UNESCO de Agua y Cultura, alojada en la Facultad de Humanidades y Ciencias de la Educación, asociada al Programa Hidrológico Internacional de UNESCO.

ఏjavier.taks@gmail.com 


\section{Referencias}

1. ARBALLO, Eduardo. Manual guía de fauna y flora de la Laguna Merín. Montevideo: PPR-MGAP, 2011.

2. AUCl - Agencia Uruguaya de Cooperación Internacional. Estado de la cooperación internacional en Uruguay. Medio Ambiente y Energía \#1. Montevideo: AUCI, 2012.

3. BAETHGEN, Walter. Gestión de riesgos climáticos para la adaptación al cambio climático en Uruguay. In: PIGNATARO, Gabriela (Ed.). Uruguay: el cambio climático aquí y ahora. Montevideo: PNÚD, 2007. p. 25-26.

4. BERTONI, Reto et al. La matriz energética: una construcción social. Montevideo: Universidad de la República: CSIC, 2010.

5. CLAES/PNUMA/DINAMA. PNUMA - Programa de Naciones Unidas para el Medio Ambiente / CLAES - Centro Latino Americano de Ecología Social / DINAMA - Dirección Nacional de Medio Ambiente. GeoUruguay 2008. Informe del estado del ambiente. Montevideo: PNUMA/CLAES, 2008.

6. GARCÍA-CARTAGENA, Martín; TORANZA, Carolina; TORRES, Maria Fernanda de. Proyectos, personas y publicaciones sobre cambio y variabilidad climática en Uruguay. In: ASTIGARRAGA, Laura; TERRA, Rafael; PICASSO, Valentín; CRUZ, Gabriela (Orgs.). Cambio y variabilidad climática: respuestas interdisciplinarias. Montevideo: UdelaR. Espacio Interdisciplinario, 2013.

7. GARCÍA-CARTAGENA, Martín; TAKS, Javier. Transferencia internacional de políticas públicas y comunidades epistémicas: el caso del proyecto de Implementación de Medidas Piloto de Adaptación al Cambio Climático en las Áreas Costeras de Uruguay. In: ASTIGARRAGA, Laura; TERRA, Rafael; CRUZ, Gabriela; PICASSO, Valentín (Coords.). Centro Interdisciplinario de Respuesta al Cambio y a la Variabilidad Climatica: vínculos ciencia-política y cienciasociedad. Montevideo: UdelaR. Espacio Interdisciplinario, 2015.

8. INGOLD, Tim. The perception of the environment. Essays in livelihood, dwelling and skill. Londres: Routledge, 2000.

9. LATCHINIÁN, Aramis. Globotomía. Del ambientalismo mediático a la burocracia ambiental. Caracas/Montevideo: Puntocero, 2011.

10. LATOUR, Bruno. Cogitamus. Seis cartas sobre las humanidades científicas. Buenos Aires: Paidós, 2012.

11. LATTANZIO, Sergio; MARRERO, Laura. Informe sobre el estado y calidad de las políticas públicas sobre cambio climático y desarrollo de Uruguay. Montevideo: Plataforma Climática Latinoamericana/Ceuta, 2012. Disponible en: https://intercambioclimatico.com/es/jce/publicaciones/12-informe-sobre-elestado-y-calidad-de-las-politicas-publicas-sobre-cambio-climatico-y-desarrollode-uruguay-2012/file.html.

12. MILTON, Kay. Introduction. Soapbox forum. Anthropological perspectives on climate change. TAJA- The Australian Journal of Anthropology, v. 19, n. 1, p. 57-88, 2008. doi: 10.1111/j.1835-9310.2008.tb00110.x 
13. MGAP- Ministerio de Ganadería, Agricultura y Pesca /DINARA- Dirección Nacional de Recursos Acuáticos. Boletín Estadístico Pesquero 2008. Montevideo: MGAP/DINARA, 2009.

14. MVOTMA - Ministerio de Vivienda, Ordenamiento Territorial y Medio Ambiente. Inventario nacional de gases de efecto invernadero. Uruguay. Montevideo: MVOTMA/SNRCC, 2017.

15. NUTTALL, Mark. Living in a world of movement: human resilience to environmental instability in Greenland. In: CRATE, Susan; NUTTALL, Mark (Eds.). Anthropology \& Climate Change. Walnut Creek: Left Coast Press, 2009. p. 292310.

16. OPP - Oficina de Planeamiento y Presupuesto/OEA- Organización de los Estados Americanos/BID - Banco Interamericano de Desarrollo. Uruguay. Estudio Ambiental Nacional. Washington DC: OEA, 1992.

17. RONCOLI, Carla; CRANE, Todd; ORLOVE, Ben. Fielding climate change in cultural anthropology. In: CRATE, Susan.; NUTTALL, Mark. (Eds). Anthropology \& Climate Change. Walnut Creek: Left Coast Press, 2009. p. 87-115.

18. SANTOS, Carlos. Fronteras líquidas: el impulso al agronegocio a partir de la nueva política de riego en Uruguay. In: Actas de la XII RAM- Reunión de Antropología del Mercosur. Misiones, Argentina, 2017.

19. TADDEI, Renzo. The politics of uncertainty and the fate of forecasters. Ethics, Policy \& Environment, v. 15, n. 2, p. 252-267, 2012. doi: 10.1080/21550085.2012.685603

20. TADDEl, Renzo. Meteorologistas e profetas da chuva. São Paulo: Terceiro Nome, 2017.

Recepción en: 26 abr 2019. Aprobación en: 01 jul 2019. 